\title{
A NOTE ON BOREL-CANTELLI LEMMAS FOR NON-UNIFORMLY HYPERBOLIC DYNAMICAL SYSTEMS
}

\author{
NICOLAI HAYDN, MATTHEW NICOL, TOMAS PERSSON, AND SANDRO VAIENTI
}

\begin{abstract}
Let $\left(B_{i}\right)$ be a sequence of measurable sets in a probability space $(X, \mathcal{B}, \mu)$ such that $\sum_{n=1}^{\infty} \mu\left(B_{i}\right)=\infty$. The classical Borel-Cantelli lemma states that if the sets $B_{i}$ are independent, then $\mu\left(\left\{x \in X: x \in B_{i}\right.\right.$ infinitely often (i.o.) $)=$ 1 .

Suppose $(T, X, \mu)$ is a dynamical system and $\left(B_{i}\right)$ is a sequence of sets in $X$. We consider whether $T^{i} x \in B_{i}$ for $\mu$ a.e. $x \in X$ and if so, is there an asymptotic estimate on the rate of entry. If $T^{i} x \in B_{i}$ infinitely often for $\mu$ a.e. $x$ we call the sequence $B_{i}$ a Borel-Cantelli sequence. If the sets $B_{i}:=B\left(p, r_{i}\right)$ are nested balls about a point $p$ then the question of whether $T^{i} x \in B_{i}$ infinitely often for $\mu$ a.e. $x$ is often called the shrinking target problem.

We show, under certain assumptions on the measure $\mu$, that for balls $B_{i}$ if $\mu\left(B_{i}\right) \geq i^{-\gamma}, 0<\gamma<1$, then a sufficiently high polynomial rate of decay of correlations for Lipschitz observations implies that the sequence is Borel-Cantelli. If $\mu\left(B_{i}\right) \geq \frac{C \log i}{i}$ then exponential decay of correlations implies that the sequence is Borel-Cantelli. If it is only assumed that $\mu\left(B_{i}\right) \geq \frac{1}{i}$ then we give conditions in terms of return time statistics which imply that for $\mu$ a.e. $p$ sequences of nested balls $B(p, 1 / i)$ are Borel-Cantelli. Corollaries of our results are that for planar dispersing billiards and Lozi maps $\mu$ a.e. $p$ sequences of nested balls $B(p, 1 / i)$ are Borel-Cantelli. We also give applications of these results to a variety of nonuniformly hyperbolic dynamical systems.
\end{abstract}

\section{INTRODUCTION}

Suppose $(X, \mathcal{B}, \mu)$ is a probability space. For a measurable set $A \subset X$, let $1_{A}$ denote the characteristic function of $A$. The classical Borel-Cantelli lemmas (see for example [11, Section 4]) state that

(1) if $\left(A_{n}\right)_{n=0}^{\infty}$ is a sequence of measurable sets in $X$ and $\sum_{n=0}^{\infty} \mu\left(A_{n}\right)<\infty$ then $\mu\left(x \in A_{n}\right.$ i.o. $)=0$.

MN and TP wish to thank the Institut Mittag-Leffler for support and hospitality. MN wishes to thank the Institut de Mathématiques Luminy also for support and hospitality. 
(2) if $\left(A_{n}\right)_{n=0}^{\infty}$ is a sequence of independent sets in $X$ and $\sum_{n=0}^{\infty} \mu\left(A_{n}\right)=\infty$, then for $\mu$ a.e. $x \in X$

$$
\frac{S_{n}(x)}{E_{n}} \rightarrow 1
$$

where $S_{n}(x)=\sum_{j=0}^{n-1} 1_{A_{j}}(x)$ and $E_{n}=\sum_{j=0}^{n-1} \mu\left(A_{j}\right)$.

Suppose now $T: X \rightarrow X$ is a measure-preserving transformation of a probability space $(X, \mu)$. If $\left(A_{n}\right)$ is a sequence of sets such that $\sum_{n} \mu\left(A_{n}\right)=\infty$ it is natural in many applications to ask whether $T^{n}(x) \in A_{n}$ for infinitely many values of $n$ for $\mu$ a.e. $x \in X$ and, if so, is there a quantitative estimate of the asymptotic number of entry times? For example, the sequence $\left(A_{n}\right)$ may be a nested sequence of balls about a point, a setting which is often called the shrinking target problem.

The property $\lim _{n \rightarrow \infty} \frac{S_{n}(x)}{E_{n}}=1$ for $\mu$ a.e. $x \in X$ is often called the Strong BorelCantelli (SBC) in contrast to the Borel-Cantelli (BC) property that $S_{n}(x)$ is unbounded for $\mu$ a.e. $x \in X$.

W. Phillipp [27] established the SBC property for sequences of intervals in the setting of certain maps of the unit interval including the $\beta$ transformation, the Gauss transformation and smooth uniformly expanding maps.

There have been some results on Borel-Cantelli lemmas for uniformly hyperbolic systems in higher dimensions. Chernov and Kleinbock [5] establish the SBC property for certain families of cylinders in the setting of topological Markov chains and for certain classes of dynamically-defined rectangles in the setting of Anosov diffeomorphisms preserving Gibbs measures. Dolgopyat [10] has related results for sequences of balls in uniformly partially hyperbolic systems preserving a measure equivalent to Lebesgue which have exponential decay of correlations with respect to Hölder observations.

Kim [21] has established the SBC property for sequences of intervals in the setting of 1-dimensional piecewise-expanding maps $T$ with $\frac{1}{\left|T^{\prime}\right|}$ of bounded variation.

Kim uses this result to prove some SBC results for non-uniformly expanding maps with an indifferent fixed point. In particular, he considers intermittent maps of the form

$$
T_{\alpha}(x)= \begin{cases}x\left(1+2^{\alpha} x^{\alpha}\right) & \text { if } 0 \leq x<\frac{1}{2} \\ 2 x-1 & \text { if } \frac{1}{2} \leq x \leq 1\end{cases}
$$

These maps are sometimes called Liverani-Saussol-Vaienti maps [22]. If $0<\alpha<1$ then $T_{\alpha}$ admits an invariant probability measure $\mu$ that is absolutely continuous with respect to Lebesgue measure $m$. We use the notation $x_{n} \sim y_{n}$ to denote $\lim _{n \rightarrow \infty} \frac{x_{n}}{y_{n}}=1$. The measure $\mu$ has an unbounded density $h(x) \sim C x^{-\alpha}$ near 0 . Kim shows that if $\left(I_{n}\right)$ is a sequence of intervals in $(d, 1]$ for some $d>0$ and $\sum_{n} \mu\left(I_{n}\right)=\infty$ then $I_{n}$ is an SBC sequence if (a) $I_{n+1} \subset I_{n}$ for all $n$ (nested intervals) 
or (b) $\alpha<(3-\sqrt{2}) / 2$. Kim shows that the condition $I_{n} \subset(d, 1]$ for some $d>0$ is in some sense optimal (with respect to the invariant measure $\mu$ ) by showing that setting $A_{n}=\left[0, n^{-1 /(1-\alpha)}\right)$ gives a sequence such that $\sum_{n} \mu\left(A_{n}\right)=\infty$ yet $T_{\alpha}^{n}(x) \in A_{n}$ for only finitely many values of $n$ for $\mu$ a.e. $x \in[0,1]$.

For the same class of maps $T_{\alpha}$, Gouëzel [13] considers Lebesgue measure $m$ (rather than the invariant probability measure $\mu$ ) and shows that if $\left(I_{n}\right)$ is a sequence of intervals such that $\sum_{n} m\left(I_{n}\right)=\infty$ then $\left(I_{n}\right)$ is a BC sequence. Assumptions (a) or (b) of Kim are not necessary for Gouëzel's result. Gouëzel uses renewal theory and obtains $\mathrm{BC}$ results but not SBC results.

At the end of this section we give an example of an intermittent type map which preserve Lebesgue measure $m$ yet for which there exists a sequence of nested intervals $I_{n}, \sum_{n} m\left(I_{n}\right)=\infty$ yet $\left(I_{n}\right)$ is not BC. Such maps may have arbitrarily high polynomial rate of decay of correlations for Hölder observations.

In the context of flows, Maucourant 25] has proved the BC property for nested balls in the setting of geodesic flows on hyperbolic manifolds of finite volume.

Recently, Gupta et al. [16] proved the SBC property for sequences of intervals in the setting of Gibbs-Markov maps and also sequences of nested balls in one-dimensional maps modeled by Young Towers. They also gave some applications to extreme value theory of deterministic systems, in particular the almost sure behavior of successive maxima of observations on such systems.

Fayad [12] has given an example of an analytic area preserving map of the threedimensional torus which is mixing of all orders, yet for which there exists a sequence of nested balls $\left(A_{n}\right)$ such that $E_{n}$ diverges yet the sequence $\left(A_{n}\right)$ is not BC.

In this short note we establish dynamical Borel-Cantelli lemmas using elementary arguments, we try to avoid dynamical assumptions beyond decay of correlations as much as we can. Our results are phrased in terms of the interplay between the measure $\mu\left(A_{n}\right)$ of the sets and the rate of decay of correlations of observations in various norms. For sets of measure $\mu\left(A_{n}\right) \leq \frac{1}{n}$ we mainly restrict to the shrinking target problem and need to make some dynamical assumptions in the guise of return time statistics. We define $E(\phi):=\int_{X} \phi d \mu$ for the expectation of an integrable observation on a dynamical system $(T, X, \mu)$ where $X$ is a metric and probability space.

In applications it is common to have decay estimates for observations on a dynamical system in various Banach space norms, for example:

(a) Bounded variation (BV) versus $L^{1}$,

$$
\left|E\left(\phi \psi \circ T^{m}\right)-E(\phi) E(\psi)\right| \leq p(m)\|\phi\|_{B V}\|\psi\|_{1},
$$

(b) Lipschitz versus $L^{\infty}$,

$$
\left|E\left(\phi \psi \circ T^{m}\right)-E(\phi) E(\psi)\right| \leq p(m)\|\phi\|_{\text {Lip }}\|\psi\|_{\infty},
$$


(c) Lipschitz versus Lipschitz,

$$
\left|E\left(\phi \psi \circ T^{m}\right)-E(\phi) E(\psi)\right| \leq p(m)\|\phi\|_{\text {Lip }}\|\psi\|_{\text {Lip }},
$$

where $p(m)$ is a rate function which tends to zero in $m$. Hölder norms are sometimes considered rather than Lipschitz but it is no essential loss to only consider Lipschitz.

In fact $B V$ versus $L^{1}$ is not so common for non-uniformly hyperbolic systems i.e. those with a stable foliation. It is implicit in Kim [21] and explicitly stated in Gupta et al. [16] that summable rate of decay (i.e. $\sum_{m} p(m)<\infty$ ) for the norms $B V$ versus $L^{1}$ implies the SBC property for any sequence of balls $B_{i}$ such that $E_{n}$ diverges.

Proposition 1.1 ([21, 16]). Suppose $(T, X, \mu)$ has summable decay of correlations with respect to $B V$ versus $L^{1}$ in the sense that for $\phi \in B V, \psi \in L^{1}(\mu)$

$$
\left|E\left(\phi \psi \circ T^{m}\right)-E(\phi) E(\psi)\right| \leq p(m)\|\phi\|_{B V}\|\psi\|_{1}
$$

where $\sum_{m} p(m)<\infty$. If $\left(B_{i}\right)$ is a sequence of balls in $X$ and $\sum_{i=0}^{\infty} \mu\left(B_{i}\right)=\infty$ then $\left(B_{i}\right)$ is $S B C$.

The proof of this result is a straightforward application of a condition for SBC by Sprindzuk [28] that we will soon state.

In this paper we will mainly consider decay of correlation of Lipschitz versus Lipschitz. The modifications of our results for Hölder versus Hölder, Lipschitz versus $L^{\infty}$ or Hölder versus $L^{\infty}$ are straightforward.

We will often use a proposition of Sprindzuk [28]:

Proposition 1.2. Let $(\Omega, \mathcal{B}, \mu)$ be a probability space and let $f_{k}(\omega),(k=1,2, \ldots)$ be a sequence of non-negative $\mu$ measurable functions and $g_{k}, h_{k}$ be sequences of real numbers such that $0 \leq g_{k} \leq h_{k} \leq 1,(k=1,2, \ldots$,$) . Suppose there exist C>0$ such that

$$
\int\left(\sum_{m<k \leq n}\left(f_{k}(\omega)-g_{k}\right)\right)^{2} d \mu \leq C \sum_{m<k \leq n} h_{k}
$$

for arbitrary integers $m<n$. Then for any $\epsilon>0$

$$
\sum_{1 \leq k \leq n} f_{k}(\omega)=\sum_{1 \leq k \leq n} g_{k}(\omega)+O\left(\theta^{1 / 2}(n) \log ^{3 / 2+\epsilon} \theta(n)\right)
$$

for $\mu$ a.e. $\omega \in \Omega$, where $\theta(n)=\sum_{1 \leq k \leq n} h_{k}$.

Example 1.0.1. Cristadoro et. al. [9] consider an intermittent map $T$ of the interval $[-1,1]$ (actually on the unit circle as 1 and -1 are identified) with an unbounded derivative at the origin. The map is implicitly defined by the equation

$$
x= \begin{cases}\left.\frac{1}{2 \gamma}(1+T(x))^{\gamma}\right)^{\gamma} & \text { if } 0 \leq x \leq \frac{1}{2 \gamma} \\ T(x)+\frac{1}{2 \gamma}(1-T(x))^{\gamma} & \text { if } \frac{1}{2 \gamma} \leq x \leq 1\end{cases}
$$


and extended as an odd function so that $T(-x)=-T(x)$. See Figure 1 .

We assume $\gamma>1$. Let $\tau=\frac{1}{\gamma-1}$ and use the notation $x_{n} \sim y_{n}$ to denote $\lim _{n \rightarrow \infty} \frac{x_{n}}{y_{n}}=1$.

The map preserves Lebesgue measure $m$ and is mixing with a polynomial rate of decay of correlations for Hölder observations. In fact

$$
\left|\int \phi \circ T^{n} \psi d m-\int \phi d m \int \psi d m\right| \leq C(\phi, \psi) n^{-\tau}
$$

for all Hölder $\phi, \psi \in L^{\infty}(m)$.

We define $T_{+}:=T_{\mid(0,1)}, T_{-}:=T_{I(-1,0)}, a_{0-}=\frac{-1}{2 \gamma}, a_{-i}=T_{-}^{-i} a_{0-}$ and $b_{i}=$ $T_{+}^{-1} a_{-(i-1)}$. Note that $T^{-1}\left(-1, a_{-n}\right)=\left(0, b_{n+1}\right) \cup\left(-1, a_{-n-1}\right)$.

It is shown in [9, Lemma 2] that $m\left(-1, a_{-n}\right) \sim(2 \gamma \tau)^{\tau} n^{-\tau}, m\left(0, b_{n}\right) \sim \frac{1}{2 \gamma}(2 \gamma \tau)^{\gamma \tau}(n-$ $1)^{-\gamma \tau}$

If we choose $\gamma>2$ then $0<\tau<1$ so $\sum_{n>0} m\left(-1, a_{-n}\right)$ diverges. Since $m\left(0, b_{n}\right) \sim$ $\frac{1}{2 \gamma}(2 \gamma \tau)^{\gamma \tau}(n-1)^{-\gamma \tau}$ and $\gamma \tau>1, \sum_{n>0} m\left(0, b_{n}\right)$ converges. The only way for the orbit of a generic point under the map $T$ to enter $\left(-1, a_{-n}\right)$ infinitely often is to enter $\left(0, b_{n}\right)$ infinitely often. Since $\sum_{n>0} m\left(0, b_{n}\right)$ converges, $m$ a.e. satisfies $T^{n} x \in\left(-1, a_{-n}\right)$ for only finitely many $n$, although $\sum_{n>0} m\left(-1, a_{-n}\right)$ diverges. This is an example of a sequence of nested sets $A_{n}:=\left(-1, a_{-n}\right)$ such that $\sum_{n>0} m\left(A_{n}\right)$ diverges yet $T^{n} x \in A_{n}$ at most finitely many times for $m$ a.e. $x$. In contradiction to the example of Kim, the map $T$ preserves Lebesgue measure rather than a measure with an unbounded density. Note also that taking $\gamma-1:=\delta>0$ the rate of decay of correlation is $C n^{-\frac{1}{\delta}}$ is at an arbitrarily high polynomial rate.

\section{Assumptions}

Suppose $(T, X, \mu)$ is an ergodic measure preserving map of a probability space $X$ which is also a metric space. We assume:

(A) For all Lipschitz functions $\phi, \psi$ on $X$ we have summable decay of correlations in the sense that there exists $C>0$, and a rate $p(k) \rightarrow \infty, \sum_{k} p(k)<\infty$ (both independent of $\phi, \psi$ ) such that

$$
\left|E\left(\phi \psi \circ T^{k}\right)-E(\phi) E(\psi)\right|<p(k)\|\phi\|_{\text {Lip }}\|\psi\|_{\text {Lip }}
$$

(B) There exists $r_{0}>0,0<\delta<1$ such that for all $p \in X$ and $\epsilon<r \leq r_{0}$

$$
\mu\{x: r<d(x, p)<r+\epsilon\}<\epsilon^{\delta} .
$$

Remark 2.0.1. If the balls $B_{i}=B\left(p, r_{i}\right),\left(r_{i} \rightarrow 0\right)$, are nested balls centered at a point $p$ then we would only require there exist $\delta(p)>0, r_{0}(p)>0$ such that $\mu\{x: r<d(x, p)<r+\epsilon\}<\epsilon^{\delta(p)}$ for all $\epsilon<r \leq r_{0}$. 


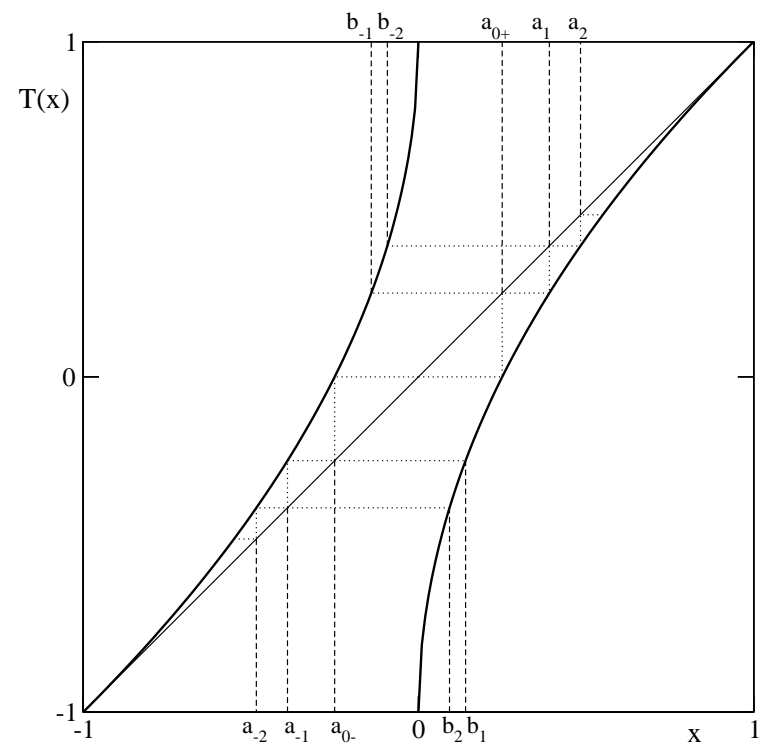

FiguRE 1. The graph of $T$.

Remark 2.0.2. We will call the sets $B_{i}$ 'balls', but our results extend to any shapes such that the indicator function of the set may be approximated closely in the $L^{1}$ norm by a Lipschitz function of reasonable Lipschitz norm, for example our results extend immediately to rectangles of bounded side ratio.

If $p(k) \leq C \alpha^{k}$ for some constants $C>0,0<\alpha<1$ then we say $T$ has exponential decay of correlations. We will also consider polynomial decay, where $p(k) \leq C k^{-m}$ for some constants $C>0, m>0$.

Our results will be formulated in terms of the measure of the balls $B_{i}$ and the rate of decay of correlation. For balls $B_{i}$ such that $\mu\left(B_{i}\right)=\frac{1}{i}$ we make additional assumptions on return time distributions and short return times, detailed in later sections.

3. Sequences of Sets $\left(B_{i}\right)$ Such that $\mu\left(B_{i}\right) \geq i^{-\gamma}$ For $0<\gamma<1$.

Define

$$
S_{n}(x)=\sum_{i=0}^{n-1} 1_{B_{k}} \circ T^{k}(x)
$$

and

$$
E_{n}=\sum_{1 \leq k \leq n} \mu\left(B_{k}\right) .
$$


Theorem 3.1. Let $0<\gamma<1$ and $\mu\left(B_{i}\right) \geq i^{-\gamma}$. Suppose $(T, X, \mu)$ satisfies (A) and (B), with $p(k) \leq C k^{-q}$. Then if $q>\frac{2 / \delta+\gamma+1}{1-\gamma}$

$$
\lim _{n \rightarrow \infty} \frac{S_{n}(x)}{E_{n}}=1
$$

for $\mu$ a.e. $x \in X$.

Remark 3.1.1. The proof of Theorem 3.1 gives the following asymptotic bounds.

$$
\sum_{i=0}^{n-1} 1_{B_{k}} \circ T^{k}(x) \approx \sum_{1 \leq k \leq n} \mu\left(B_{k}\right)+O\left(\theta^{1 / 2+\epsilon}(n) \log ^{3 / 2+\epsilon} \theta(n)\right)
$$

for $\mu$ a.e. $x \in X$, where $\theta(n)=\sum_{1 \leq k \leq n} \mu\left(B_{k}\right)$.

Remark 3.1.2. We note that if $\mu\left(B_{i}\right)=\frac{1}{i}$ then no degree of polynomial decay in the Lipschitz norm ensures that the sequence $\left(B_{i}\right)$ is $\mathrm{BC}$, even if the $B_{i}$ are nested balls. To see this consider the intermittent map example of Kim [21], $T_{\alpha}:[0,1] \rightarrow$ $[0,1]$ with the balls $B_{i}:=\left[0, i^{-1 /(1-\alpha)}\right)$ so that $\mu\left(B_{i}\right) \equiv \frac{1}{i}$. The density $h(x)=\frac{d \mu}{d m}$ behaves like $x^{-\alpha}$ near 0 and the rate of decay of correlations with respect to Lipschitz versus $L^{\infty}$ functions is $n^{1-\frac{1}{\alpha}}$. The constant $\delta$ in the statement of the theorem (from Property $(B))$ may be taken as $1-\alpha$ by observing $\int_{0}^{\epsilon} x^{-\alpha} d x \leq C \epsilon^{1-\alpha}$. As $\alpha \rightarrow 0$, $\delta(\alpha) \rightarrow 1$ and $q(\alpha) \rightarrow \infty$ yet for each $0<\alpha<1$, the sequence $B_{i}=\left[0, i^{-1 /(1-\alpha)}\right)$ is not BC.

Proof. For each $k$ let $\tilde{f}_{k}$ be a Lipschitz function such that $\tilde{f}_{k}(x)=1_{B_{k}}(x)$ if $x \in B_{k}$, $\tilde{f}_{k}(x)=0$ if $d\left(B_{k}, x\right)>\left(k(\log k)^{2}\right)^{-1 / \delta}, 0 \leq \tilde{f}_{k} \leq 1$ and $\left\|\tilde{f}_{k}\right\|_{\text {Lip }} \leq\left(k(\log k)^{2}\right)^{1 / \delta}$. Clearly we may construct such functions by linear interpolation of $1_{B_{k}}$ and 0 on a region $r \leq d\left(p_{k}, x\right) \leq r+\left(k(\log k)^{2}\right)^{-1 / \delta}$.

In the proposition above we will take $f_{k}=\tilde{f}_{k} \circ T^{k}(x), g_{k}=E\left(\tilde{f}_{k}\right)$, where $E(\phi)=$ $\int \phi d \mu$ for any integrable function $\phi \in L^{1}(\mu)$. The constants $h_{k}$ will be chosen later.

Note that for $\mu$ a.e. $x \in \Omega, f_{i}(x)=1_{B_{i}}\left(T^{i} x\right)$ except for finitely many $i$ by the Borel-Cantelli lemma as $\mu\left(x: f_{i}(x) \neq 1_{B_{i}}\left(T^{i} x\right)\right)=\mu\left(x: r_{i}<d\left(T^{i} x, p_{i}\right)<r_{i}+\right.$ $\left.\left(i(\log i)^{2}\right)^{-1 / \delta}\right)<\left(i(\log i)^{2}\right)^{-1}$ by assumption (B). Furthermore $\sum_{k} \mu\left(B_{k}\right)=\sum_{k} g_{k}+$ $O(1)$.

A rearrangement of terms in (囝) means that it suffices to show that there exists a $C>0$ such that

$$
\sum_{i=m}^{n} \sum_{j=i+1}^{n} E\left(f_{i} f_{j}\right)-E\left(f_{i}\right) E\left(f_{j}\right) \leq C \sum_{i=m}^{n} h_{i}
$$

for arbitrary integers $n>m$ (where $h_{i}$ will be chosen later). 
We split each sum $\sum_{j=i+1}^{n} E\left(f_{i} f_{j}\right)-E\left(f_{i}\right) E\left(f_{j}\right)$ into the terms

$$
I_{i}=\sum_{j=i+1}^{i+\Delta} E\left(f_{i} f_{j}\right)-E\left(f_{i}\right) E\left(f_{j}\right)
$$

and

$$
I I_{i}=\sum_{j=i+\Delta+1}^{n} E\left(f_{i} f_{j}\right)-E\left(f_{i}\right) E\left(f_{j}\right)
$$

where here we put $\Delta=\left[i^{\sigma}\right]$ with the value of $0<\sigma<1$ is to be chosen later.

The first term $I_{i}$ is roughly estimated by

$$
\sum_{j=i+1}^{i+i^{\sigma}} E\left(f_{i} f_{j}\right)-E\left(f_{i}\right) E\left(f_{j}\right) \leq \sum_{j=i+1}^{i+i^{\sigma}} E\left(f_{i} f_{j}\right) \leq \sum_{j=i+1}^{i+i^{\sigma}} \frac{2}{j^{\gamma}} \leq i^{\sigma} \frac{2}{i^{\gamma}} .
$$

The second term $I I_{i}$ we bound using the decay of correlations

$$
\begin{aligned}
I_{i} & =\sum_{j=i+i^{\sigma}}^{\infty} E\left(f_{i} f_{j}\right)-E\left(f_{i}\right) E\left(f_{j}\right) \\
& =\sum_{j=i+i^{\sigma}}^{\infty} \int_{X} f_{i}\left(T^{i} x\right) f_{j}\left(T^{j} x\right) d \mu-E\left(f_{i}\right) E\left(f_{j}\right) \\
& =\sum_{j=i+i^{\sigma}}^{\infty} \int_{X} f_{i}(x) f_{j}\left(T^{j-i} x\right) d \mu-E\left(f_{i}\right) E\left(f_{j}\right) \\
& \leq \sum_{j=i+i^{\sigma}}^{\infty}\left\|f_{i}\right\|_{\operatorname{Lip}}\left\|f_{j}\right\|_{\operatorname{Lip}} p(j-i) \\
& \leq \sum_{\beta=1}^{\infty} C\left[\left(i+i^{\sigma}+\beta\right)\left(\log \left(i+i^{\sigma}+\beta\right)\right)^{2}\right]^{1 / \delta}\left[i(\log i)^{2}\right]^{1 / \delta}\left(i^{\sigma}+\beta\right)^{-q} .
\end{aligned}
$$

If $\sigma<1-\gamma$ then $\left(I_{i}\right)$ is bounded by $C i^{1-2 \gamma-\rho}$ for some $\rho>0$. If $q \sigma>\frac{2}{\delta}+\gamma+1$ then $\left(I I_{i}\right)$ is bounded by const. $i^{-\gamma}$. Solving for $\sigma<1-\gamma$ and then for $q$ we see that if $q>\frac{2 / \delta+\gamma+1}{1-\gamma}$ then $\sum_{j=i+1} \int E\left(f_{i} f_{j}\right)-E\left(f_{i}\right) E\left(f_{j}\right) \leq C \max \left\{i^{1-2 \gamma-\rho}, i^{-\gamma}\right\}$.

In Sprindzuk's theorem we now take $h_{i}=C \max \left\{i^{1-2 \gamma-\rho}, i^{-\gamma}\right\}$. With this choice of $h_{i}$ we have $\theta(n)^{1 / 2} \leq C \max \left\{n^{(1-\gamma) / 2}, n^{1-\gamma-\rho}\right\}$. This gives the Strong BorelCantelli property as the error term $O\left(\theta^{1 / 2}(n) \log ^{3 / 2+\epsilon} \theta(n)\right)$ is negligible with respect to $\sum_{1 \leq k \leq n} g_{k}=\sum_{1 \leq k \leq n} E\left(f_{k}\right) \simeq C n^{1-\gamma}$. 
4. Return time distributions And Sequences $\left(B_{i}\right) \operatorname{SUCH}$ That $\mu\left(B_{i}\right) \geq \frac{\log i}{i}$

We now consider the case $\mu\left(B_{i}\right) \geq \frac{\log i}{i}$. In this case we assume exponential decay of correlations.

Theorem 4.1. Suppose $\mu\left(B_{i}\right) \geq \frac{\log i}{i}$ and $(T, X, \mu)$ satisfies $(A)$ and $(B)$. Then if $(T, X, \mu)$ has exponential decay of correlations

$$
\lim _{n \rightarrow \infty} \frac{S_{n}(x)}{E_{n}}=1
$$

for $\mu$ a.e. $x \in X$.

Proof. Let $p(k) \leq C \alpha^{k}$ for some $0<\alpha<1$. In this setting we again split $\sum_{j=i+1}^{n} E\left(f_{i} f_{j}\right)-$ $E\left(f_{i}\right) E\left(f_{j}\right)$ as above in two sums $I_{i}$ and $I I_{i}$ where this time $\Delta=\left[(\log i)^{\sigma}\right]$, where $\sigma>1$ will be chosen below. We obtain

$$
I_{i}=\sum_{j=i+1}^{i+(\log i)^{\sigma}} \frac{\log j}{j} \leq(\log i)^{\sigma} \frac{\log i}{i}=\frac{(\log i)^{\sigma+1}}{i}
$$

and, using the decay of correlations,

$$
\begin{aligned}
I I_{i} & =\sum_{j=i+(\log i)^{\sigma}}^{N} \int_{X} f_{i}\left(T^{i} x\right) f_{j}\left(T^{j} x\right) d \mu-E\left(f_{i}\right) E\left(f_{j}\right) \\
& \leq C \sum_{\beta=1}^{\infty} \alpha^{(\log i)^{\sigma}+\beta}\left((\log i)^{\sigma}+i+\beta\right)^{2 / \delta} i^{2 / \delta}
\end{aligned}
$$

As $\sigma>1$ for large enough $i$ the series above is bounded by $\frac{\log i}{i}$. We take $1<$ $\sigma<2$ and $h_{k}=(\log k)^{\sigma+1} / k$. Since $\sigma<2$ the error $O\left(\theta(n)^{1 / 2} \log ^{3 / 2+\epsilon} \theta(n)\right)$ is negligible with respect to $\sum_{k=1}^{n} g_{k} \sim \sum_{k=1}^{n} \frac{\log k}{k} \sim \frac{1}{2}(\log n)^{2}$ as $\theta(n)=\sum_{k=1}^{n} h_{k} \sim$ $\sum_{k=1}^{n} \frac{(\log k)^{\sigma+1}}{k} \sim(\log n)^{\sigma+2}$.

4.1. Applications of Theorem 3.1 and Theorem 4.1. We now list some dynamical systems which satisfy assumptions $(A)$ and $(B)$. Recall that although we have called the sets $B_{i}$ balls, any sets $B_{i}$ which are geometrically regular in the sense that their indicator function may be approximated in the $L^{1}$ norm by a Lipschitz function of reasonable Lipschitz norm (for example rectangles) also satisfy the conclusions of Theorem 3.1 and Theorem 4.1.

Dispersing billiard systems: satisfy assumption (B) (as the invariant measure is equivalent to Lebesgue) and hence our results apply to these systems if they have sufficiently high rates of decay of correlations. The class of dispersing billiards considered by Young [29] and Chernov [4] have exponential decay of correlations. 
Lozi maps: it is shown in Gupta et al. [15] that assumption (B) is satisfied by a broad class of Lozi mappings. Lozi mappings have exponential decay of correlations. Compact group extensions of Anosov systems: Dolgopyat [10 has shown that compact group extensions of Anosov diffeomorphisms are typically rapid mixing i.e. have superpolynomial decay of correlations. These systems satisfy also Assumption B as they are volume preserving.

Interval maps: one-dimensional non-uniformly expanding maps with an absolutely continuous invariant probabiility measure $\mu$ and density $h=\frac{d \mu}{d m} \in L^{1+\delta}(m)$ for some $\delta>0$ satisfy condition (A) and (B). For example the class of maps considered by Collet [7].

\section{Sequences of BAlls $\left(B_{i}\right)$ Such that $\mu\left(B_{i}\right) \leq i^{-1}$ AND $\sum_{i} \mu\left(B_{i}\right)=\infty$.}

If the measure of the balls $B_{i}$ satisfies $\mu\left(B_{i}\right) \leq i^{-1}$ then our arguments based solely on decay of correlations break down and to obtain results we make more assumptionsin particular we focus on the case of nested balls $B_{i+1} \subset B_{i}$ and make assumptions on return time statistics.

In this section we focus on the shrinking target problem in which $B_{i}(p)=B\left(p, r_{i}\right)$ the ball of radius $r_{i}$ about $p$ about a point $p \in X$ such that $\mu\left(B\left(p, r_{i}\right)\right)=i^{-1}$. Our proof and results generalize with no change to the setting where there exists a constant $C>0$ such that $r_{i+1}<C r_{i}$ and $\mu\left(B\left(p, r_{i}\right)\right) \geq \frac{C}{i}$.

We first observe, as remarked in Fayad [12], that the set of points $G$ such that $T^{i} x \in B_{i}(p)$ for infinitely many $i$ has measure zero or one. This follows as $T^{i+1} x \in$ $B_{i+1}(p)$ implies that $T^{i}(T x) \in B_{i}(p)$ and hence $G$ is $T$ invariant. If $(T, X, \mu)$ is ergodic this implies that $\mu(G)=0$ or $\mu(G)=1$.

5.1. Return time distributions. We now show how a return time distribution implies Borel-Cantelli lemmas in certain settings. Fix $p \in X$ and set $B_{i}=B\left(p, r_{i}\right)$ where $r_{i} \rightarrow 0$ so that $B_{i}$ is a sequence of nested balls with center $p$. Let $\tau_{B_{i}}$ be a random variable defined on the set $B_{i}=B\left(p, r_{i}\right)$ which gives the first return time i.e. $\tau_{B_{i}}(x)=\inf \left\{n \geq 1: T^{n}(x) \in B_{i}\right\}$. We define the conditional probability measure $\mu_{B_{r}}$ on $B_{r}$ by $\mu_{B_{r}}(A) ;=\frac{\mu\left(A \cap B_{r}\right)}{\mu\left(B_{r}\right)}$.

For many dynamical systems distributional limit laws for return time statistics have been proven, of the form: for $\mu$ a.e. $p$, if $B_{r}=B(p, r)$ for $r>0$ then

$$
\lim _{r \rightarrow 0} \mu_{B_{r}}\left\{y \in B_{r}: \tau_{B_{r}} \mu\left(B_{r}\right)<t\right\}=F(t)
$$

where $F(t)$ is a distribution function. Commonly $F(t)=1-e^{-t}$, an exponential law.

Let $S_{n}=\sum_{j=0}^{n-1} 1_{B_{j}}, E_{n}=\sum_{j=0}^{n-1} \mu\left(B_{j}\right)$ with $\lim _{n \rightarrow \infty} E_{n}=\infty$. We now state a simple lemma. 
Lemma 5.1. Suppose that $B_{i}$ is a sequence of balls (not necessarily nested). If $E\left(S_{n}-E_{n}\right)^{2} \leq g(n)\left(E_{n}\right)^{2}$ for a sequence $g(n)$ such that $\lim _{n \rightarrow \infty} g(n)=0$ then $\lim \sup \frac{S_{n}(x)}{E_{n}} \geq 1$ for $\mu$ a.e. $x \in X$.

Proof. The assumptions imply that $E\left[\left(\frac{S_{n}}{E_{n}}-1\right)^{2}\right] \leq g(n)$. Hence $E\left[\left|\frac{S_{n}}{E_{n}}-1\right|>\epsilon\right] \leq$ $\frac{E\left(S_{n}-E_{n}\right)^{2}}{\epsilon^{2}} \leq \frac{g(n)}{\epsilon^{2}}$ by Chebyshev's inequality. If we take a subsequence $n_{k}$ such that $\sum_{k} g\left(n_{k}\right)<\infty$, then by Borel-Cantelli for $\mu$ a.e. $x \in X,\left|\frac{S_{n}(x)}{E_{n}}-1\right|>\epsilon$ for only finitely many $n_{k}$. Let $G_{\epsilon}=\left\{x:\left|\frac{S_{n}(x)}{E_{n}}-1\right|>\epsilon i\right.$.o. $\}$, then $\mu\left(G_{\epsilon}^{c}\right)=1$. Taking a countable sequence $\epsilon_{m}=\frac{1}{m}$ and noting $\mu\left(\cap_{m} G_{\epsilon_{m}}^{c}\right)=1$ implies the result.

In fact if the $B_{i}$ are nested and $(T, X, \mu)$ is ergodic then the assumptions of the lemma above may be weakened to

Lemma 5.2. Suppose that $B_{i}$ is a nested sequence of balls and $(T, X, \mu)$ is ergodic. If $E\left(S_{n}-E_{n}\right)^{2}<\eta\left(E_{n}\right)^{2}$ for some $\eta<1$ then $T^{n}(x) \in B_{n}$ infinitely often for $\mu$ a.e. $x \in X$.

Proof. Let $G$ be the set of points $x$ such that $T^{i} x \in B_{i}$ for infinitely many $i$. Then $\mu(G)=1$ or $\mu(G)=0$. We assume $\mu(G)=0$ and derive a contradiction.

$$
\eta>\int_{X}\left(\frac{S_{n}(x)}{E_{n}}-1\right)^{2} d \mu \geq \int_{G^{c}}\left(\frac{S_{n}(x)}{E_{n}}-1\right)^{2} d \mu
$$

Let $A_{N}:=\left\{x \in G^{c}: T^{i}(x) \notin B_{i}\right.$ for all $\left.i \geq N\right\}$. Then

$$
\eta>\int_{G^{c}}\left(\frac{S_{n}(x)}{E_{n}}-1\right)^{2} d \mu \geq \int_{A_{N}}\left(\frac{S_{n}(x)}{E_{n}}-1\right)^{2} d \mu
$$

But $\lim _{n \rightarrow \infty} \int_{A_{N}}\left(\frac{S_{n}(x)}{E_{n}}-1\right)^{2} d \mu=\mu\left(A_{N}\right)$. Hence $\eta>\mu\left(A_{N}\right)$ for all $N$, a contradiction.

We now state our main result of this section. Let $B_{i}(p)$ be a decreasing sequence of balls about a point $p, S_{n}=\sum_{j=0}^{n-1} 1_{B_{j}}$ and $E_{n}=\sum_{j=0}^{n-1} \mu\left(B_{j}\right)$.

Theorem 5.1. Suppose that $(T, X, \mu)$ has exponential decay of correlations, that property $(B)$ holds, that $B_{i}=B\left(p, r_{i}\right)$ for some point $p$ and $\mu\left(B_{i}\right) \geq i^{-1}$. Also assume that

$$
\lim _{i \rightarrow \infty} \mu_{B_{i}}\left\{y \in B_{i}: \tau_{B_{i}} \mu\left(B_{i}\right)<t\right\}=F(t)
$$

for some distribution function $F(t)$ such that $\lim _{t \rightarrow 0^{+}} F(t)=0$. Then for $\mu$ a.e. $x \in X$

$$
\limsup \frac{S_{n}(x)}{E_{n}} \geq 1 \text {. }
$$


Proof. We choose $c \geq 0$ so that for all $i$

$$
(*) \sum_{\beta=1}^{\infty} C(i+c \log i+\beta)^{2 / \delta} i^{2 / \delta} \alpha^{c \log i+\beta} \leq i^{-2} .
$$

Since $B_{j} \subset B_{i}$ for $j>i$

$$
\sum_{j=i}^{i+c \log i} \mu\left(B_{i} \cap T^{-(j-i)} B_{j}\right) \leq \sum_{j=i}^{i+c \log i} \mu\left(B_{i} \cap T^{-(j-i)} B_{i}\right) .
$$

Since $\lim _{t \rightarrow 0} F(t)=0$, given $\eta>0$ there exists $t^{*}$ such that $F(t) c<\eta$ for all $0<t \leq t^{*}$. As

$$
\lim _{i \rightarrow \infty} \mu_{B_{i}}\left\{y \in B_{i}: \tau_{B_{i}} \mu\left(B_{i}\right)<t\right\}=F(t)
$$

there exists a number $n^{*}$ such that for $i \geq n^{*}$

$$
\mu_{B_{i}}\left\{y \in B_{i}: \tau_{B_{i}}<-c \log \mu\left(B_{i}\right)\right\} \leq F\left(t^{*}\right)
$$

Hence for $i>n^{*}$

$$
\sum_{j=i}^{i+c \log i} \mu\left(B_{i} \cap T^{-(j-i)} B_{j}\right) \leq \sum_{j=i}^{i+c \log i} \mu\left(B_{i} \cap T^{-(j-i)} B_{i}\right) \leq F\left(t^{*}\right) \frac{c \log i}{i} .
$$

Recalling that

$$
E\left[\left(S_{n}-E_{n}\right)^{2}\right]=2 \sum_{i=1}^{n} \sum_{j>i}\left(\mu\left(B_{i} \cap T^{-(j-i)} B_{j}\right)-\mu\left(B_{i}\right) \mu\left(B_{j}\right)\right)+\sum_{i=1}^{n}\left(\mu\left(B_{i}\right)-\mu\left(B_{i}\right)^{2}\right)
$$

we note first that $\sum_{i=1}^{n}\left(\mu\left(B_{i}\right)-\mu\left(B_{i}\right)^{2}\right) \leq E_{n}$ and write

$$
\begin{gathered}
\sum_{i=1}^{n} \sum_{j>i}\left(\mu\left(B_{i} \cap T^{-(j-i)} B_{j}\right)-\mu\left(B_{i}\right) \mu\left(B_{j}\right)\right)=\sum_{n \geq i>n^{*}} \sum_{j>i}\left(\mu\left(B_{i} \cap T^{-(j-i)} B_{j}\right)-\mu\left(B_{i}\right) \mu\left(B_{j}\right)\right) \\
+\sum_{1 \leq i<n^{*}} \sum_{j>i}\left(\mu\left(B_{i} \cap T^{-(j-i)} B_{j}\right)-\mu\left(B_{i}\right) \mu\left(B_{j}\right)\right)
\end{gathered}
$$

The term $\sum_{1 \leq i<n^{*}} \sum_{j>i}\left(\mu\left(B_{i} \cap T^{-(j-i)} B_{j}\right)-\mu\left(B_{i}\right) \mu\left(B_{j}\right)\right)$ we bound by $n^{*} E_{n}$ while

$$
\begin{gathered}
\sum_{n \geq i>n^{*}} \sum_{j>i}\left(\mu\left(B_{i} \cap T^{-(j-i)} B_{j}\right)-\mu\left(B_{i}\right) \mu\left(B_{j}\right)\right) \\
\leq \sum_{i=n^{*}}^{n} c F\left(t^{*}\right) \frac{\log i}{i}+\sum_{i=n^{*}}^{n} \sum_{j=i+c \log i}^{n}\left(\mu\left(B_{i} \cap T^{-(j-i)} B_{j}\right)-\mu\left(B_{i}\right) \mu\left(B_{j}\right)\right) \\
\leq \frac{\eta}{2}(\log n)^{2}+K
\end{gathered}
$$


where we have used to the estimate $(*)$ to bound the second term by a constant $K$ . Thus for $n>n^{*}, E\left[\left(S_{n}-E_{n}\right)^{2}\right]<\eta E_{n}^{2}+2 K+n^{*} E_{n}$. Since $\eta$ was arbitrary and $E_{n}$ diverges this implies that given $\epsilon>0$ there exists an $N$ such that for all $n>N$, $E\left[\left(S_{n}-E_{n}\right)^{2}\right]<\epsilon E_{n}^{2}$. Thus Lemma 5.1 implies the result.

5.2. Applications of Theorem 5.1. We now give a brief list of systems, beside Axiom A [18], which have been shown to have the property that for $\mu$ a.e. $p$ if $B_{i}=B\left(p, r_{i}\right)$ then

$$
\lim _{r_{i} \rightarrow 0}\left(\mu_{B_{i}}\right)^{-1}\left\{y \in B_{i}: \tau_{B_{i}} \mu\left(B_{i}\right)<t\right\}=F(t)
$$

for a distribution function $F(t)$ such that $\lim _{t \rightarrow 0} F(t)=0$. In the examples below $F(t)=1-e^{-t}$, an exponential law. We abbreviate this property by saying that the system has an exponential law for first return times to balls (recall this holds only for $\mu$ a.e. point).

Dispersing billiard systems: These systems satisfy assumption (B) (as the invariant measure is equivalent to Lebesgue). The class of dispersing billiards considered by Young [29] and Chernov [4] have exponential decay of correlations. An exponential law for first return times to balls has been established by Gupta et al. [15] (and a Poisson distribution for further visits to balls by Chazottes and Collet [3]).

Lozi maps [8, 26]: it is shown in Gupta et al. [15] that assumption (B) and an exponential return time law is satisfied by a broad class of Lozi mappings (which have exponential decay of correlations).

Compact group extensions of Anosov systems: Dolgopyat [10] has shown that compact group extensions of Anosov diffeomorphisms are typically rapid mixing i.e. have superpolynomial decay of correlations. These systems satisfy also Assumption (B) as they are volume preserving. Gupta [14] has shown the existence of an exponential law for the first return times to nested balls if the system is rapidly mixing.

Interval maps: one-dimensional non-uniformly expanding maps with an absolutely continuous invariant probabiility measure $\mu$ and density $h=\frac{d \mu}{d m} \in L^{1+\delta}(m)$ for some $\delta>0$ satisfy condition (A) and (B). The class of maps considered by Collet [7] have been shown to have an exponential law for first return times to balls.

\section{Short Return times And Sequences $\left(B_{i}\right)$ Such that $\mu\left(B_{i}\right) \geq(i \log i)^{-1}$}

In the theory of return time statistics and extreme value theory a crucial role is played by short return times. Recent research has established that for certain chaotic dynamical systems short returns are rare in the sense that we call property $(\mathrm{C})$ :

(C) for $\mu$ a.e. $p \in X$ there exists $\eta>0$ and $k>1$ such that for all $i$ sufficiently large

$$
\mu\left(B_{i}(p) \cap T^{-r} B_{i}(p)\right) \leq i^{-1-\eta}
$$


for all $r=1, \ldots, \log ^{k}(i)$.

The property $(\mathrm{C})$ is a form of non-recurrence which implies certain limit laws in return time statistics and extreme value theory, to our knowledge first proved in the setting of non-uniformly expanding maps by Collet [7]. It has been verified for various systems, for example Sinai dispersing billiards and Lozi mappings [15]. We give the proof of Property (C) for Sinai dispersing billiards as an appendix. Beside Axiom A systems, dynamical systems for which Property $(\mathrm{C})$ have been established include:

(1) Billiard maps for dispersing billiards without cusps: for example the systems described in [29, 4, 6]. For a proof of this property see the Appendix.

(2) Lozi maps [8, 26]: it is shown in Gupta et al. [15] that assumption (C) is satisfied by a broad class of Lozi mappings. Gupta [14] shows the same for toral extensions of certain non-uniformly and uniformly expanding intervals maps.

(3) Certain one-dimensional maps: Collet first established Property (C) in the setting of 1-d non-uniformly expanding maps with acip with exponential decay of correlations [7]. These results have been generalized to a broad class of $1 \mathrm{~d}$ maps, including Lorenz like maps [15, 19].

Theorem 6.1. Suppose $(T, X, \mu)$ has exponential decay of correlations and satisfies conditions (B) and $(C)$. Then for $\mu$ a.e. $p \in X$ if $B_{i}=B\left(p, r_{i}\right)$ is a sequence of decreasing balls about $p$ with $\mu\left(B_{i}\right) \geq(i \log i)^{-1}$ then

$$
\lim _{n \rightarrow \infty} \frac{S_{n}(x)}{E_{n}}=1
$$

for $\mu$ a.e. $x \in X$, where as before $S_{n}=\sum_{j=0}^{n-1} 1_{B_{i}}$ and $E_{n}=E\left(S_{n}\right)=\sum_{j=0}^{n-1} \mu\left(B_{j}\right)$

Corollary 6.1. If $(T, X, \mu)$ is a Sinai dispersing billiard map (see Appendix for precise description) or a Lozi map (see [15]) then in the notation of Theorem 6.1 for $\mu$ a.e. $p \in X$ if $B_{i}$ is a sequence of decreasing balls about $p$ with $\mu\left(B_{i}\right) \geq(i \log i)^{-1}$ then

$$
\lim _{n \rightarrow \infty} \frac{S_{n}(x)}{E_{n}}=1
$$

for $\mu$ a.e. $x \in X$.

Proof of Corollary 6.1. The proof that Property (C) holds for Sinai dispersing billiard maps with $k=5$ is given in the Appendix. It is a very slight modification of the proof in Gupta et al. [15] where the sequence $\mu\left(B_{i}\right)=i^{-1}$ was considered. A similar straightforward modification of the proof of the corresponding result for sequences $\left(B_{i}\right)$ such that $\mu\left(B_{i}\right)=i^{-1}$ given in Gupta et al. [15] in the setting of Lozi maps establishes the conclusions of the corollary for Lozi maps. 
BOREL-CANTELLI LEMMAS FOR NON-UNIFORMLY HYP. DYN. SYS.

Proof of Theorem 6.1. We consider the case $\mu\left(B_{i}\right)=(i \log i)^{-1}$, which implies the corresponding result for $\mu\left(B_{i}\right) \geq(i \log i)^{-1}$. We define $f_{k}$ and $g_{k}$ as before and split up $\sum_{j=i+1}^{n} E\left(f_{i} f_{j}\right)-E\left(f_{i}\right) E\left(f_{j}\right)$ into the terms $I_{i}$ and $I I_{i}$ with $\Delta=\left[\log ^{2} i\right]$. A rough estimate yields

$$
\begin{aligned}
I_{i} & =\sum_{j=i+1}^{i+\log ^{2} i} E\left(f_{i} f_{j}\right)-E\left(f_{i}\right) E\left(f_{j}\right) \\
& \leq \sum_{j=i+1}^{i+\log ^{2} i} E\left(f_{i} f_{j}\right) \\
& \leq C\left(\log ^{2} i\right)(i \log i)^{-1} \\
& \leq C i^{-1-(\eta / 2)}
\end{aligned}
$$

for large $i$ and any positive $\eta$. For the second sum we obtain

$$
\begin{aligned}
I I_{i} & =\sum_{j=i+\log ^{2} i}^{n} E\left(f_{i} f_{j}\right)-E\left(f_{i}\right) E\left(f_{j}\right) \\
& \leq \sum_{\beta=1}^{\infty} C i^{2 / \delta}\left(i+\log ^{2} i+\beta\right)^{2 / \delta} \alpha^{\log ^{2} i+\beta} \\
& \leq C(i \log i)^{-1}
\end{aligned}
$$

for sufficiently large $i$. As above in light of Proposition 1.2 this implies the conclusion of the theorem.

\section{Discussion.}

There are several questions that are prompted by this work. Here are some that we have considered but not resolved as yet.

(1) If $(T, X, \mu)$ is a smooth dynamical system with acip and positive metric entropy is it true that for $\mu$ a.e. $p$ if $B_{i}=B\left(p, r_{i}\right)$ is a nested sequence of balls about $p$ then

$$
\lim _{r_{i} \rightarrow 0}\left(\mu_{B_{i}}\right)^{-1}\left\{y \in B_{i}: \tau_{B_{i}} \mu\left(B_{i}\right)<t\right\}=F_{p}(t)
$$

for a distribution function $F_{p}(t)$ such that $\lim _{t \rightarrow 0} F_{p}(t)=0$ ?

(2) Chazottes and Collet [3] have shown that if $(T, X, \mu)$ is a dynamical system modeled by a Young tower, with exponential decay of correlations and a one-dimensional unstable foliation then for $\mu$ a.e. point $p$, if $B_{i}=B\left(p, r_{i}\right)$ is a nested sequence of balls about $p$ then

$$
\lim _{r_{i} \rightarrow 0}\left(\mu_{B_{i}}\right)^{-1}\left\{y \in B_{i}: \tau_{B_{i}} \mu\left(B_{i}\right)<t\right\}=1-e^{-t}
$$


In fact they have shown much more, including a Poisson law for multiple returns. If such systems satisfied Property (B) (respectively Property (C)) then the conclusion of Theorem 5.1 (respectively Theorem 6.1) would hold. Does Property (B) or (C) hold for $\mu$ a.e. point in such systems?

(3) Is there an example of a smooth volume preserving dynamical system $(T, X, \mu)$ which has exponential decay of correlations yet there is a sequence of balls $B_{i}$, $\mu\left(B_{i}\right) \geq i^{-1}$ which is not Borel-Cantelli?

7.1. Appendix: Property C for Planar Dispersing Billiard Maps. We first describe the class of billiards for which we can prove Property C. For a good general reference to billiards see [6].

Let $\Gamma=\left\{\Gamma_{i}, i=1, \ldots, k\right\}$ be a family of pairwise disjoint, simply connected $C^{3}$ curves with strictly positive curvature on the two-dimensional torus $\mathbb{T}^{2}$. The billiard flow $B_{t}$ is the dynamical system generated by the motion of a point particle in $Q=\mathbb{T}^{2} /\left(\cup_{i=1}^{k}\left(\right.\right.$ interior $\left.\Gamma_{i}\right)$ with constant unit velocity inside $Q$ and with elastic reflections at $\partial Q=\cup_{i=1}^{k} \Gamma_{i}$, where elastic means "angle of incidence equals angle of reflection". If each $\Gamma_{i}$ is a circle then this system is called a periodic Lorentz gas. The billiard flow is Hamiltonian and preserves a probability measure (which is Liouville measure) $\tilde{\mu}$ given by $d \tilde{\mu}=C_{Q} d q d t$ where $C_{Q}$ is a normalizing constant and $q \in Q$, $t \in \mathbb{R}$ are Euclidean coordinates.

We first consider the billiard map $T: \partial Q \rightarrow \partial Q$. Let $r$ be a one-dimensional co-ordinatization of $\Gamma$ corresponding to length and let $n(r)$ be the outward normal to $\Gamma$ at the point $r$. For each $r \in \Gamma$ we consider the tangent space at $r$ consisting of unit vectors $v$ such that $(n(r), v) \geq 0$. We identify each such unit vector $v$ with an angle $\theta \in[-\pi / 2, \pi / 2]$. The boundary $M$ is then parametrized by $M:=\partial Q=$ $\Gamma \times[-\pi / 2, \pi / 2]$ so that $M$ consists of the points $(r, \theta) . T: M \rightarrow M$ is the Poincaré map that gives the position and angle $T(r, \theta)=\left(r_{1}, \theta_{1}\right)$ after a point $(r, \theta)$ flows under $B_{t}$ and collides again with $M$, according to the rule angle of incidence equals angle of reflection. Thus if $(r, \theta)$ is the time of flight before collision $T(r, \theta)=B_{h(r, \theta)}(r, \theta)$. The billiard map preserves a measure $d \mu=c_{M} \cos \theta d r d \theta$ equivalent to 2-dimensional Lebesgue measure $d m=d r d \theta$ with density $\rho(x)$ where $x=(r, \theta)$.

We say that the billiard map and flows satisfies the finite horizon condition if the time of flight $h(r, \theta)$ is bounded above. A good reference for background results for this section are the papers [1, 2, 29, 4].

It is known (see [4, Lemma 7.1] for finite horizon and [4, Section 8] for infinite horizon) that dispersing billiard maps expand in the unstable direction in the Euclidean metric $|\cdot|=\sqrt{(d r)^{2}+(d \phi)^{2}}$, in that $\left|D T_{u}^{n} v\right| \geq C \tilde{\lambda}^{n}|v|$ for some constants $C, \tilde{\lambda}>1$ which is independent of $v$. In fact $\left|L_{n}\right| \geq C \tilde{\lambda}^{n}\left|L_{0}\right|$ where $L_{0}$ is a segment of unstable manifold (once again in the Euclidean metric) and $L_{n}$ is $T^{n} L_{0}$. 
We choose $N_{0}$ so that $\lambda:=C \tilde{\lambda}^{N_{0}}>1$ and then $T^{N_{0}}$ (or $D T^{N_{0}}$ ) expands unstable manifolds (tangent vectors to unstable manifolds) uniformly in the Euclidean metric.

It is common to use the $p$-metric in proving ergodic properties of billiards. Young uses this semi-metric in [29]. Recall that for any curve $\gamma$, the $p$-norm of a tangent vector to $\gamma$ is given as $|v|_{p}=\cos \phi(r)|d r|$ where $\gamma$ is parametrized in the $(r, \phi)$ plane as $(r, \phi(r))$. The Euclidean metric in the $(r, \phi)$ plane is given by $d s^{2}=d r^{2}+d \phi^{2}$; this implies that $|v|_{p} \leq \cos \phi(r) d s \leq d s=|v|$. We will use $l_{p}(C)$ to denote the length of a curve in the $p$-metric and $l(C)$ to denote length in the Euclidean metric. If $\gamma$ is a local unstable manifold or local stable manifold then $C_{1} l(\gamma)_{p} \leq l(\gamma) \leq C_{2} \sqrt{l_{p}(\gamma)}$.

For planar dispersing billiards there exists an invariant measure $\mu$ (which is equivalent to 2-dimensional Lebesgue measure) and through $\mu$ a.e. point $x$ there exists a local stable manifold $W_{l o c}^{s}(x)$ and a local unstable manifold $W_{l o c}^{u}(x)$. The SRB measure $\mu$ has absolutely continuous (with respect to Lebesgue measure ) conditional measures $\mu_{x}$ on each $W_{l o c}^{u}(x)$. The expansion by $D T$ is unbounded however in the $p$-metric at $\cos \theta=0$ and this may lead to quite different expansion rates at different points on $W_{l o c}^{u}(x)$. To overcome this effect and obtain uniform estimates on the densities of conditional SRB measure it is common to define homogeneous local unstable and local stable manifolds. This is the approach adopted in [1, 2, 4, 29]. Fix a large $k_{0}$ and define for $k>k_{0}$

$$
\begin{gathered}
I_{k}=\left\{(r, \theta): \frac{\pi}{2}-k^{-2}<\theta<\frac{\pi}{2}-(k+1)^{-2}\right\} \\
I_{-k}=\left\{(r, \theta):-\frac{\pi}{2}+(k+1)^{-2}<\theta<-\frac{\pi}{2}+k^{-2}\right\}
\end{gathered}
$$

and

$$
I_{k_{0}}=\left\{(r, \theta):-\frac{\pi}{2}+k_{0}^{-2}<\theta<\frac{\pi}{2}-k_{0}^{-2}\right\} .
$$

In our setting we call a local unstable (stable) manifold $W_{l o c}^{u}(x),\left(W_{l o c}^{s}(x)\right)$ homogeneous if for all $n \geq 0 T^{n} W_{l o c}^{u}(x)\left(T^{-n} W_{l o c}^{s}(x)\right)$ does not intersect any of the line segments in $\cup_{k>k_{0}}\left(I_{k} \cup I_{-k}\right) \cup I_{k_{0}}$. Homogeneous $W_{l o c}^{u}(x)$ have almost constant conditional SRB densities $\frac{d \mu_{x}}{d m_{x}}$ in the sense that there exists $C>0$ such that $\frac{1}{C} \leq \frac{d \mu_{x}\left(z_{1}\right)}{d m_{x}} / \frac{d \mu_{x}\left(z_{2}\right)}{d m_{x}} \leq C$ for all $z_{1}, z_{2} \in W_{l o c}^{u}(x)$ (see [4, Section 2] and the remarks following Theorem 3.1).

From this point on all the local unstable (stable) manifolds that we consider will be homogeneous. Bunimovich et al. [2, Appendix 2, Equation A2.1] give quantitative estimates on the length of homogeneous $W_{l o c}^{u}(x)$. They show there exists $C, \tau>0$ such that $\mu\left\{x: l\left(W_{l o c}^{s}(x)\right)<\epsilon\right.$ or $\left.l\left(W_{l o c}^{u}(x)\right)<\epsilon\right\} \leq C \epsilon^{\tau}$ where $l(C)$ denotes 1dimensional Lebesgue measure or length of a rectifiable curve $C$. In our setting $\tau$ could be taken to be $\frac{2}{9}$, its exact value will play no role but for simplicity in the forthcoming estimates we assume $0<\tau<\frac{1}{2}$. 
The natural measure $\mu$ has absolutely continuous conditional measures $\mu_{x}$ on local unstable manifolds $W_{l o c}^{u}(x)$ which have almost uniform densities with respect to Lebesgue measure on $W_{l o c}^{u}(x)$ by [4, Equation 2.4].

Let $A_{\sqrt{\epsilon}}=\left\{x:\left|W_{l o c}^{u}(x)\right|>\sqrt{\epsilon}\right\}$ then $\mu\left(A_{\sqrt{\epsilon}}^{c}\right)<C \epsilon^{\tau / 2}$. Let $x \in A_{\sqrt{\epsilon}}$ and consider $W_{l o c}^{u}(x)$. Since $\left|T^{-k} W_{l o c}^{u}(x)\right|<\lambda^{-1}\left|W_{l o c}^{u}(x)\right|$ for $k>N_{0}$ the optimal way for points $T^{-k}(y)$ in $T^{-k} W_{l o c}^{u}(x)$ to be close to their preimages $y \in W_{l o c}^{u}(x)$ is for $T^{-k} W_{l o c}^{u}(x)$ to overlay $W_{l o c}^{u}(x)$, in which case it has a fixed point and it is easy to see $l\left\{y \in W_{\text {loc }}^{u}(x): d\left(y, T^{-k} y\right)<\epsilon\right\} \leq l\left\{y \in \mathbb{R}: d\left(y, \frac{y}{\lambda}\right)<\epsilon\right\} \leq\left(1-\lambda^{-1}\right) \epsilon$. Accordingly $l\left\{y \in W_{l o c}^{u}(x): d\left(y, T^{-k} y\right)<\epsilon\right\} \leq C \sqrt{\epsilon} l\left\{y \in W_{l o c}^{u}(x)\right\}$. Recalling that the density of the conditional SRB-measure $\mu_{x}$ is bounded above and below with respect to one-dimensional Lebesgue measure we obtain $\mu_{x}\left(A_{\sqrt{\epsilon}}^{c}\right)<C \sqrt{\epsilon}$. Integrating over all unstable manifolds in $A_{\sqrt{\epsilon}}$ (throwing away the set $\mu\left(A_{\sqrt{\epsilon}}^{c}\right)$ ) we have $\mu\left\{x: d\left(T^{-k} x, x\right)<\epsilon\right)<C \epsilon^{\tau / 2}$. Since $\mu$ is $T$-invariant $\mu\left\{x: d\left(T^{k} x, x\right)<\epsilon\right\}<C \epsilon^{\tau / 2}$ for $k>N_{0}$. Hence for any iterate $T^{k}, k>N_{0}$

$$
\mathcal{E}_{k}(\epsilon):=\mu\left\{x: d\left(T^{k} x, x\right)<\epsilon\right\}<C \epsilon^{\tau / 2} .
$$

Define

$$
E_{k}:=\left\{x: d\left(T^{j} x, x\right) \leq \frac{2}{\sqrt{k}} \text { for some } 1 \leq j \leq(\log k)^{5}\right\} .
$$

We have shown that for any $\delta>0$, for all sufficiently large $k, \mu\left(E_{k}\right) \leq k^{-\tau / 4+\delta}$. For simplicity we take $\mu\left(E_{k}\right) \leq k^{-\sigma}$ where $\sigma<\tau / 4-\delta$.

Define the Hardy-Littlewood maximal function $M_{l}$ for $\phi(x)=1_{E_{l}}(x) \rho(x)$ where $\rho(x)=\frac{d \mu}{d m}(x)$, so that

$$
M_{l}(x):=\sup _{a>0} \frac{1}{m\left(B_{a}(x)\right)} \int_{B_{a}(x)} 1_{E_{l}}(y) \rho(y) d m(y) .
$$

A theorem of Hardy and Littlewood [23, Theorem 2.19] implies that

$$
m\left(\left|M_{l}\right|>C\right) \leq \frac{\left\|1_{E_{l}} \rho\right\|_{1}}{C}
$$

where $\|\cdot\|_{1}$ is the $L^{1}$ norm with respect to $m$. Let

$$
F_{k}:=\left\{x: \mu\left(B_{k^{-\gamma / 2}}(x) \cap E_{k^{\gamma / 2}}\right) \geq\left(k^{-\gamma \beta / 2}\right) k^{\gamma / 2} .\right.
$$

Then $F_{k} \subset\left\{M_{k^{\gamma / 2}}>k^{-\gamma \beta / 2}\right\}$ and hence

$$
m\left(F_{k}\right) \leq \mu\left(E_{k^{\gamma / 2}}\right) k^{\gamma \beta / 2} \leq C k^{-\gamma \sigma} k^{\gamma \beta / 2} .
$$

If we take $0<\beta<\sigma$ and $\gamma>\sigma / 2$ then for some $\delta>0, k^{-\gamma \sigma} k^{\gamma \beta / 2}<k^{-1-\delta}$ and hence

$$
\sum_{k} m\left(F_{k}\right)<\infty \text {. }
$$


Thus for $m$ a.e. (hence $\mu$ a.e.) $x_{0} \in X$ there exists $N\left(x_{0}\right)$ such that $x_{0} \notin F_{k}$ for all $k>N\left(x_{0}\right)$. Thus along the subsequence $n_{k}=k^{-\gamma / 2}, \mu\left(B_{n_{k}}\left(x_{0}\right) \cap T^{-j} B_{n_{k}}\left(x_{0}\right)\right) \leq$ $n_{k}^{-1-\delta}$ for $k>N\left(x_{0}\right)$. This is sufficient to obtain an estimate along the subsequence $(n \log n)^{-1}$. Since $\lim _{k \rightarrow \infty}\left(\frac{k+1}{k}\right)^{\gamma / 2}=1$ if $k^{\gamma / 2} \leq n \log n \leq(k+1)^{\gamma / 2}$ then for sufficiently large $n \mu\left(B_{(n \log n)^{-1}}\left(x_{0}\right) \cap T^{-j} B_{(n \log n)^{-1}}\left(x_{0}\right)\right) \leq \mu\left(B_{n_{k}}\left(x_{0}\right) \cap T^{-j} B_{n_{k}}\left(x_{0}\right)\right) \leq$ $n_{k}^{-1-\delta} \leq 2(n \log n)^{-1-\delta}$. As $\frac{d \mu}{d m}(p)$ is finite for $\mu$ a.e. $p$ this implies the result for $\mu\left(B_{i}\right)=i \log i$ by the Lebesgue density theorem. We now control the iterates $1 \leq j \leq N_{0}$. If $x_{0}$ is not periodic then $\min _{1 \leq i<j \leq N_{0}} d\left(T^{i} x_{0}, T^{j} x_{0}\right) \geq s\left(x_{0}\right)>0$ and hence for large enough $n$, for all $1 \leq j \leq N_{0}, \mu\left(B_{n^{-1}}\left(x_{0}\right) \cap T^{-j} B_{n^{-1}}\left(x_{0}\right)\right)=0$.

\section{REFERENCES}

[1] L. A. Bunimovich, Ya. G. Sinai and N. I. Chernov, Markov partitions for two-dimensional hyperbolic billiards, Russian Math. Surveys, 45:3, 1990, 105-152.

[2] L. A. Bunimovich, Ya. G. Sinai and N. I. Chernov, Statistical properties of two-dimensional hyperbolic billiards, Russian Math. Surveys, 46, 1991, 47-106.

[3] J. R. Chazottes and P. Collet. Poisson approximation for the number of visits to balls in nonuniformly hyperbolic dynamical systems, Preprint.

[4] N. Chernov. Decay of correlations in dispersing billiards, Journal of Statistical Physics, 94 (1999), 513-556.

[5] N. Chernov and D. Kleinbock. Dynamical Borel-Cantelli lemmas for Gibbs measures, Israel J. Math. 122 (2001), 1-27.

[6] N. Chernov and R. Markarian. Chaotic Billiards, Mathematical surveys and monographs, Vol 127, (2006), American Mathematical Society.

[7] P. Collet. Statistics of closest return for some non-uniformly hyperbolic systems, Erg. Th. \& Dyn. Syst., 21 (2001), 401-420.

[8] P. Collet and Y. Levy, Ergodic properties of the Lozi Mappings, Commum. Math. Phys. 93 (1984), 461-481.

[9] G. Cristadoro, N. Haydn, P. Marie and S. Vaienti, Statistical properties of intermittent maps with unbounded derivative, preprint.

[10] D. Dolgopyat. Limit theorems for partially hyperbolic systems, Trans. AMS 356 (2004) 16371689.

[11] R. Durrett. Probability: Theory and Examples, Second Edition, Duxbury Press, 2004.

[12] B. Fayad, Two remarks on the shrinking target property, arXiv:math/0501205v1.

[13] S. Gouëzel, A Borel-Cantelli lemma for intermittent interval maps, Nonlinearity, 20 (2007), no. 6, 1491-1497.

[14] C. Gupta, Extreme Value Distributions for some classes of Non-Uniformly Partially Hyperbolic Dynamical Systems, Ergodic Theory and Dynamical Systems, 30 (2010), 757-771.

[15] C. Gupta, M. Holland and M. Nicol, Extreme value theory and return time statistics for dispersing billiard maps and flows, Lozi maps and Lorenz-like maps, to appear in Erg. Th. Dyn. Syst.

[16] C. Gupta, M. Nicol and W. Ott, A Borel-Cantelli lemma for non-uniformly expanding dynamical systems, to appear in Nonlinearity.

[17] N. Haydn, Y. Lacroix and S. Vaienti, Hitting and return time statistics in ergodic dynamical systems, Ann. Probab., 33, (2205), 2043-2050. 
[18] M. Hirata, Poisson Limit Law for Axiom-A diffeomorphisms, Erg. Thy. Dyn. Sys., 13 (1993), $533-556$.

[19] M. P. Holland, and M. Nicol and A. Török, Extreme value distributions for non-uniformly hyperbolic dynamical systems, To appear in Transactions AMS.

[20] A. Katok and J. M. Streleyn, Invariant manifolds, entropy and billiards; smooth maps with singularities, Springer Lecture Notes in Math. 1222, (1986).

[21] D. Kim, The dynamical Borel-Cantelli lemma for interval maps, Discrete Contin. Dyn. Syst. 17 (2007), no. 4, 891-900.

[22] C. Liverani, B. Saussol and S. Vaienti, A probabilistic approach to intermittency, Ergodic Theory Dynam. Systems 19 (1999), no. 3, 671-685.

[23] P. Mattila. Geometry of sets and measures in Euclidean spaces: fractals and rectifiability. Cambridge Studies in Advanced Mathematics, 44, 1995.

[24] I. Melbourne and M. Nicol, Large deviations for nonuniformly hyperbolic systems, Transactions of the AMS, 360, (2008) 6661-6676.

[25] F. Maucourant, Dynamical Borel-Cantelli lemma for hyperbolic spaces, Israel J. Math., 152 (2006), 143-155.

[26] M. Misiurewisz, Strange attractors for the Lozi Mappings, Non Linear Dynamics, R.G. Helleman (ed), New York, The New York Academy of Sciences 1980.

[27] W. Phillipp, Some metrical theorems in number theory, Pacific J. Math. 20 (1967) 109-127.

[28] Vladimir G. Sprindzuk, Metric theory of Diophantine approximations, V. H. Winston and Sons, Washington, D.C., 1979, Translated from the Russian and edited by Richard A. Silverman, With a foreword by Donald J. Newman, Scripta Series in Mathematics. MR MR548467 (80k:10048).

[29] L.-S. Young. Statistical properties of dynamical systems with some hyperbolicity, Ann. of Math. 147 (1998) 585-650.

Nicolai Haydn, Department of Mathematics, University of Southern California, Los ANGeles, CA, 90089

E-mail address: nhaydn@usc.edu

Matthew Nicol, Department of Mathematics, University of Houston, Houston, TX, 77204-3008

E-mail address: nicol@math.uh.edu

Tomas Persson, Department of Mathematics, Lunds Tekniska Högskola, Box 118, 22100 LUND

E-mail address: tomasp@maths.1th.se

Sandro Vaienti, UMr-6207 Centre de Physique Théorique, CNRS, Universités D'Aix-Marseille I, II, Université du Sud, Toulon-Var and FRUMAM, FÉdéderation de Recherche des Unités de Mathématiques de Marseille, CPT, Luminy Case 907, F-13288 Marseille Cedex 9, France

E-mail address: vaienti@cpt.univ-mrs.fr 